\title{
Delivering focused ultrasound to intervertebral discs using time-reversal
}

S. Qiao', D. Elbes', O. Boubriak', J.P.G Urban'², C-C. Coussios,' R.O. Cleveland"

'Institute of Biomedical Engineering, Department of Engineering Science, University of Oxford, UK

Department of Physiology, Anatomy \& Genetics, University of Oxford, UK

Address correspondence to:

Robin Cleveland,

Institute of Biomedical Engineering

Department of Engineering Science

Old Road Campus Research Building

Oxford OX3 7DQ

UK

Email: robin.cleveland@eng.ox.ac.uk 


\section{Abstract}

2 Chronic low back pain causes more disability world-wide than any other condition, and is thought to 3 arise in part through loss of biomechanical function of degenerate intervertebral discs (IVD). Current 4 treatments can involve replacing part or all of the degenerate IVD by invasive surgery. Our vision is 5 to develop a minimally invasive approach in which high intensity focused ultrasound (HIFU) is used 6 to mechanically fractionating degenerate tissue in an IVD; a fine needle is then used to first remove 7 the fractionated tissue and then inject a biomaterial able to restore normal physiological function. The 8 goal of this manuscript is to demonstrate the feasibility of trans-spinal HIFU delivery using 9 simulations of three-dimensional ultrasound propagation in models derived from patient CT scans. 10 The CT data were segmented in to bone, fat and other soft tissue for three patients. Ultrasound arrays 11 were placed around the waist of each patient model and time-reversal was used to determine the source signals necessary to create a focus in the centre of the disc. The simulations showed that for

$13 \quad 0.5 \mathrm{MHz}$ ultrasound a focus could be created in most of the lumbar IVDs, with the pressure focal gain 14 ranging from 3.2 to 13.7 . In conclusion, it is shown that, with patient specific planning, focusing 15 ultrasound into an IVD is possible in the majority of cases despite the complex acoustic path 16 introduced by the bony structures of the spine.

17 Keywords: Chronic low back pain; trans-spinal HIFU; full wave modelling; Time-reversal 


\section{INTRODUCTION}

20 Chronic low back pain is one of the most prevalent musculoskeletal conditions with $84 \%$ of adults suffering low back pain at some points in their lives(Shaheed et al. 2014; Walker 2000). It causes more disability worldwide than any other condition (Hoy et al. 2014) and has huge economic costs, both through health-care consumption, and lost productivity(Maniadakis \& Gray 2000). Although its causes are not well understood, many cases of low back pain are associated with degeneration of the intervertebral disc (Cheung et al. 2009), which loses its load-bearing role and hence may place inappropriate stresses on other spinal structures. The only treatments available to patients who fail conservative treatments are surgical interventions, such as replacement of the degenerate disc by a disc prosthesis. These surgical interventions are not ideal as they have relatively high complications associated with them and the effectiveness of outcome is rather variable(Willems et al. 2013). Less invasive alternatives for early disc degeneration such as partial disc replacement (PDR) have thus been developed (Coric \& Mummaneni 2008; Lewis 2012). In PDR, the gelatinous core of the degenerated disc, called the nucleus pulposus (see Fig. 1), is replaced by an implant, however current methods of removing the original degraded material require a surgical procedure and also leave an opening in the outer annulus of the disc through which implanted material can be expelled (Di Martino et al. 2005; Coric \& Mummaneni 2008). To significantly reduce the risk of expulsion, we envision an ultrasound-based, minimally-invasive PDR procedure where the degenerate nucleus would be fractionated by high frequency focussed ultrasound and the fractionated material removed via a fine gauge needle inserted through the annulus of the disc into the disc nucleus. Physiologically appropriate injectable biomaterials could then be inserted into the evacuated nucleus using the same needle.

The fractionation would be effected by high intensity focused ultrasound (HIFU) which has been employed for many indications, including: treatment of solid tumours, thrombolysis, drug delivery and neurological disorders(Al-Bataineh et al. 2012; Medel et al. 2012). In the majority of HIFU applications, thermally ablated lesions are formed as energy absorption at the ultrasound focus results 
in heating at a rate on the order of $1 \cdot \mathrm{C} / \mathrm{s}$ (ter Haar \& Coussios 2007). The principle of thermal therapy has been applied for the treatment of low back pain with an interstitial ultrasound device (Nau et al. 2007), with the aim of producing thermal necrosis of ingrowing nociceptive nerve fibres for coagulation and restructuring of annular collagen. An MR guided HIFU system was adapted for thermally treating facet joint osteoarthritis pain in a phase I study(Weeks et al. 2012). However, thermal based therapies, which produce coagulated tissue that is normally mechanically stiff, are likely not the best approach here where the goal is to remove the tissue. Thermal therapies also have the potential for heat to conduction to adjacent tissue resulting in unwanted side effects, e.g., the nerve root and vertebral end plates, in spine applications (Brown et al. 2015; Forslund et al. 2006). A more promising approach is to use short ultrasound pulses with high amplitude but low average power, that result in intense cavitation which will mechanical disrupt tissue, by a process referred to as histotripsy (Lake et al. 2008; Roberts et al. 2006; Vlaisavljevich et al. 2013; Xu et al. 2008) or boiling histotripsy (Canney et al. 2010; Khokhlova et al. 2011). Once the degenerate material is fractionated it would be removed using a fine gauge needle and then a biocompatible material injected to restore disc function.

One barrier to employing ultrasound in the IVD is that the treatment site is deeply situated in a complex anatomy with bony structures and multiple tissue layers in the acoustic path. This means the ultrasound propagation will be significantly distorted and so generating tightly focused beams will be challenging. Numerical studies investigating the use of interstitial ultrasound thermal ablation of IVD and vertebral and spinal tumors have been reported (Persson et al. 2005; Scott et al. 2014) and demonstrated the possibility of deliver ultrasound into spinal structures in a minimal-invasive way despite the complex environment. However, extracorporeal delivery is yet more challenging. A similar problem has been encountered in the transcranial and transcostal ultrasound delivery and timereversal techniques have been shown to be effective at restoring a focus in these environments (Aubry et al. 2008; Fink 1992; Fink et al. 2003). A key limitation in using time-reversal is that an acoustic transducer is needed at the target location in order to determine the propagation delays associated with acoustic path to the source. Strategies to accomplish this include: placing a hydrophone at the target (Pernot et al. 2007; Thomas \& Fink 1996), using numerical simulations to compute the phase delays 
(Marquet et al. 2009; Aubry et al. 2008; Robertson et al. 2017) and using cavitation bubble emissions as a source (Gateau et al. 2010). Transcranial focusing using time-reversal has been shown to have millemetre accuracy in cadavar models (Marsac et al. 2012).

The goal of this paper was to use numerical simulations to demonstrate that it is feasible to focus ultrasound into an intervertebral discs in humans using an extracorporeal source. Three-dimensional models of the torsos of three patients (small, medium and large) were derived from clinical CT scans. Ray tracing and numerical time-reversal were employed to determine the design of an appropriate array configuration. It was shown that by correcting for aberrations induced by the tissue inhomogeneities an appropriate ultrasound focus can be formed at the target location in the centre of most lumbar IVDs. The predicted pressure amplitudes are likely not sufficient to induce inertial cavitation in the IVD, and suggest that it will be necessary to introduce a cavitation nucleation into the disc to make mechanical fractionation of the nucleus of the disc feasible.

\section{MATERIALS AND METHODS}

Human data sets were derived from anonymised kidney-ureter-bladder (KUB) CT scans with a 0.625 $\mathrm{mm}$ slice thickness and $0.625 \mathrm{~mm}$ in-plane pixel size. The anonymised images were acquired as part of standard-of-care treatment and IRB approval was not required for their use in this research. The scans covered the anatomical structures from the vertebral body L1 to the pelvis. The CT data were imported into Matlab (R2014a, Mathworks Inc., Natick, MA, USA) and segmented into air, softtissue, fat and bone using thresholds on the Hounsfield units. In some slices the cortical bone around the vertebral bodies was so thin that it did not automatically segment into bone and in these cases the segmentation was manually corrected. The segmentation of soft-tissue into two types: fat and other soft-tissue was deemed an acceptable approximation from the point of view of acoustic propagation as most soft-tissue has similar acoustic properties with the exception of fat. The physical parameters used in the simulations are given in Table I. After segmentation, the processed CT images were 
stacked to form a 3D model that was loaded into the acoustic simulation package. Figure 2(a)

98 illustrates the steps involved in the segmentation and acoustic simulation process.

In order to investigate the effect of the size and inhomogeneous nature of patients on the focal gain and targeting accuracy of ultrasound, CT scans from three patients were segmented, see Fig 2(b-d). They will be referred to as model I (small), model II (medium), and model III (large). The circumferences of these models were $789 \mathrm{~mm}, 929 \mathrm{~mm}$ and $1129 \mathrm{~mm}$ respectively, which for European males correspond to a BMI of 20, 2940 respectively (Smith \& Haslam 2007) where the mean BMI for a male in the UK is 28.7 (Johnson et al. 2014). The lateral widths were $290 \mathrm{~mm}, 320$ $\mathrm{mm}$ and $380 \mathrm{~mm}$ respectively. Because there is the possibility of degeneration for every lumbar disc, the four discs between the vertebral bodies L1 through L5 were all treated as potential targets for HIFU. It was not possible to automatically segment the IVD from the CT images as the Hounsfield units of the IVD were very similar to the surrounding soft tissue. Instead it was assumed that the soft tissue between two consecutive vertebrae was the IVD; the outline of the vertebrae was superimposed on the soft tissue from which the IVD boundary was determined. This was done for visualisation and targeting only and for the simulations the properties of the disc were taken to be those of soft tissue.

The ultrasound simulation was carried out using PZFlex (Weidlinger Associates Inc., Mountain View, CA, USA) which employs the finite element method and an explicit time-domain algorithm to solve locally linearised versions of the continuity, momentum and state equations. Hexahedral elements with 8 nodes were used for regularly meshing of the model. The code was run for linear propagation and the grid was chosen with 15 elements per wavelength which we have previously found gives sufficient accuracy at an acceptable computational cost (Qiao et al. 2016). All the simulation results were post-processed and visualised in Matlab.

The size of the computational domain varied for the different patients and the target IVD. On average the size of the domain was $100 \mathrm{~mm} \times 370 \mathrm{~mm} \times 175 \mathrm{~mm}$ and captured the whole targeted IVD and the adjacent vertebral bodies. Due to the limited PC memory, only the posterior part of patient was included in the model which resulted in grid size of $500 \times 1850 \times 875$ elements for a total memory 
requirement in excess of $64 \mathrm{~GB}$. Neglecting the anterior portion of the patient was considered appropriate for the application in question, as the presence of gas in the bowel means that a reliable acoustic window does not exist and so placement of transducers on the stomach of the patient was not considered. In addition, no elements were place in a region 160 200 mm wide around the centre-line of the patient in order to protect the spinal cord from direct insonation by the array.

In order to apply time-reversal, a point source generating a $500 \mathrm{kHz}$ sinusoidal waveform was located at the centre of the IVD, and the pressure waveforms on a spherically curved surface outside the patient body were recorded. From this simulation two appropriate acoustic windows, on either flank of the patient, were identified. A ray tracing method was used to confirm the placement of the array. In each acoustic window an ultrasound array was generated that consisted of 192 rectangular elements each of dimensions $5 \mathrm{~mm} \times 3 \mathrm{~mm}$ tightly arranged in 3 rows of 64 . The transmit signals for each element was obtained by time-reversing the recorded waveforms from the simulation with a point source.

\section{RESULTS}

Figure 3 compares representative results for model II from ray tracing and a full-wave acoustic simulation. In Fig 3 (a) the rays that originated from the target location and were not obstructed by the bone structures on the way to the skin are shown with yellow lines. These rays were then extended to a spherical surface with the origin at the target and the radius of $170 \mathrm{~mm}$ to find the possible acoustic window for the extracorporeal ultrasound delivery, which is indicated by the red contours in Figure 3. The pressure distribution on the same spherical surface from the acoustic simulation with a point source is superimposed on the image and it can be seen that the high pressure area from the PZFlex simulations falls within the boundaries given by the ray-tracing method. However because the simulation also accounts for refraction and absorption in the tissue there are regions of low pressure even at locations where ray theory suggests sound is present. Therefore ray tracing was used to determine the initial placement of the ultrasound array for a specific disc. This was followed by full acoustic simulations of the back-propagation from the target to the surface of the transducer and then 
time-reversal was employed to determine the transmit waveforms. Finally, a full acoustic forward

150 simulation was used to determine the pressure field generated in the IVD.

151

152

Figure 4 shows the simulation results for ultrasound delivery to the L4L5 IVD, that is, the disc between vertebrae L4 and L5, of model II where the source condition was either uniform excitation (which would result in focusing in water) or time-reversed signals. The white contours represent the vertebral bones and the black contours, the estimated region of the IVD. The pressure gain is calculated by normalising the field pressure by the source pressure. As will be presented in the discussion, a gain in excess of 20 would be needed to generate cavitation in native disc tissue but if one introduces nucleation agents a gain in excess of 2 should be sufficient.

It can be seen that the uniform excitation (Fig 4(a)) failed to produce focusing in the disc and the pressure distribution was severely distorted by the vertebral bone and the multiple soft tissue interfaces resulting in local "hot-spots" at the edge of the disc that extended out into the surrounding tissue. These are likely due to a combination of scattering from the vertebral bodies and refraction from variations in the soft tissue path. In comparison using the time-reversed signals (Fig 4(b)), resulted in an ultrasound focus with a pressure gain of around 9 at the intended location (indicated by the white star). The focal spot actually consists of three lobes, a main lobe and two similar amplitude side lobes. This is a feature of the interference between the two crossed beams and was also observed in simulation is water (data not shown). In addition, to the high pressure in the target region, there were also "hot spots" at the edge of the disc and in the surrounding tissue but the key difference is that the highest pressure is now in the target focal area. An approach to ensure fractionation only occurs in the target location is described in the discussion.

Simulations were carried out for all four lumbar IVDs in all three patient models. The pressure distributions in the cross-section planes cutting through the centre of the discs are presented in Figure 5 with the black contours representing the estimated region of the IVD. In most cases, the ultrasound beams coming from the two lateral sides are clearly observed, and a focus is formed at the interaction of the two ultrasound beams in the centre of the IVD. The interference of the two ultrasound beams 
resulted in the three lobe focal structure seen in Fig 4(b) although in some cases, e.g., L3L4 in model II, the distortion resulted in only two lobes. In many cases there are "hot spots" at the edge of the discs with a similar or even higher pressure gain than the value of the focus. The only disc which showed no focusing was L4L5 in model III (the largest patient) where little of the disc experienced a pressure gain of greater than unity. This was likely due to interference from the pelvis and the longer propagation path in the patient. In addition, for L3L4 in model III, although a focus was generated at the target location there was a region of higher pressure gain at the edge of the disc. Across all three patient models the L1L2 disc showed the greatest disturbance of the field with significant regions of tissue outside the disc having high pressure amplitudes. This is likely due to the interference from the rib cage which reduces the effective acoustic window to the disc.

In each case the pressure gain and the offset of the peak pressure from the desired target location (distance between target location and location of the peak pressure) were extracted from the simulation results. Figure 6 shows the results for the pressure gain and focal position offset for 11 of the 12 cases; the one exception being the lowest lumbar disc L4L5 of model III in which a focus was not formed. It can be seen that the focal gain was highest for model I (range 7.0 to 13.7) and lowest for model III ( $<1$ to 4.5$)$. The most likely reason is that for larger patients the propagation path in the soft tissue is longer and therefore the sound suffers from more attenuation; an effect that cannot be overcome by time-reversal. For models I and II the pressure gained exceeded 5.9 for all four lumbar. The location of the focus (Fig 6(b)) was formed within $1.5 \mathrm{~mm}$ of the intended target for all 11 cases, with a range of offsets varying from $0.28 \mathrm{~mm}$ to $1.47 \mathrm{~mm}$. In general, model I and II had offsets of less than $0.6 \mathrm{~mm}$. One notable exception was L1L2 for model I which had the highest offset but we note that in Fig 6(a) it was also the case which produced the lowest gain for model I. As described above, it appears that the presence of the rib cage in the propagation path to L1L2 resulted in poorer focusing, potentially because the rib cage is closer to the focus in the smaller patient and therefore more of a solid angle is perturbed.

The pressure distributions were normalised to the pressure gain at the focus in each case and the $-3 \mathrm{~dB}$ and $-6 \mathrm{~dB}$ contours are shown in Figure 7 . Since the internal structure of the disc cannot be 
distinguished from the CT image, the disc nucleus (the desired target region for the application) is approximated as an ellipse $(22 \mathrm{~mm} \times 16 \mathrm{~mm})$ indicated by the thin black contours. It can be seen that there is a $-3 \mathrm{~dB}$ region in the target region of the nucleus but, as described above, there are also regions in the annulus that are at $-3 \mathrm{~dB}$ in all but one case. The L1L2 disc, in which ribs are present in the propagation path, appeared to be most challenging to generate a tight focus in, with $-3 \mathrm{~dB}$ contours crossing from the annulus to the nucleus in all three cases. The $-6 \mathrm{~dB}$ contours extend from the outside of the disc to the centre of the nucleus in almost every case. These results suggest that even though a focus was created in the target region the distribution of the field and the limited pressure gains mean that using ultrasound to create localised fractionation of the disc in the centre of the nucleus is likely not possible, however an approach to overcome this is presented in the discussion.

\section{DISCUSSION}

In this numerical study, three 3-D human spine models were extracted from clinical CT scans, and a time-reversal technique was employed to deliver ultrasound into the nucleus of the lumbar IVDs. The simulation results suggest that, with the help of time-reversal to compensate for the aberration in the propagation path, ultrasound can be focused in the nucleus of an IVD with focal pressure gain of 3.2 or better. Although time-reversal has been successfully used in transcranial and trans-costal ultrasound delivery to minimize the effect of the bone structure on the ultrasound focus, we believe this is first time in which the effectiveness of time-reversal has been demonstrated in trans-spinal ultrasound delivery. Delivery to the IVD is a particularly challenging as both the vertebral body and the complex spinal processes significantly distort the ultrasound beam. For example, in trans-costal application, ray-tracing has been employed to compensate for the presence of ribs to turn off individual elements that are blocked by the ribs (Bobkova et al. 2010; Qiao et al. 2013). But for this study, even though ray-tracing was helpful in identifying an appropriate window the phase information was not sufficient to correct for aberrations presumably because the bone is close to the focal region and therefore scattering and diffraction associated with the bone strongly impacts the acoustic field in the focal region. 
Flank paths were chosen as acoustic windows to the IVD, recall Fig 3, in order to avoid gas in the bowel and to prevent the ultrasound beams from passing through the spinal cord; this placement should also mean that beams avoid vascular structures such as the aorta. The ability to focus using time-reversal was investigated in all four lumbar discs in each of the three different size patients. The simulation results indicate that the discs that would best candidates for ultrasound therapy are L2L3 and L3L4 where for all three patients the $-3 \mathrm{~dB}$ focal region was restricted to a volume less than $6 \mathrm{~mm}$ in diameter in the centre of the nucleus; although there were lobes at $-3 \mathrm{~dB}$ in the annulus of the discs too. For the lowermost disc, L4L5, the ilium has the potential to block the ultrasound beam coming from the posterolateral sides and limit the area of the acoustic window. However, the spinal anatomy varies between patients and for the small and medium patients time-reversal still resulted in successful ultrasound focusing. For L1L2 there were a number of "hot spots" away from the target location which appeared to be due to interference from the ribs. These result suggest that lumbar discs are potential targets for ultrasound therapy but that detailed patient-specific pre-treatment calculations are needed in order to identify whether an IVD is a good target for ultrasound therapy and the placement and phasing of an array source.

Our vision for how therapeutic ultrasound would work in an IVD is that cavitation would fractionate the nucleus of the disc with minimal heating. Although the simulations did demonstrate the ability to focus in the nucleus neither the pressure amplitudes nor the shape of the focal region were appropriate to create localised cavitation in vivo. First, the amplitudes were too low; assuming a typical maximum source level of $1 \mathrm{MPa}$ pressure amplitudes could be as low as $3.2 \mathrm{MPa}$ whereas mechanical tissue disintegration requires peak negative pressures in excess of $20 \mathrm{MPa}$ in order have a consistent dense cavitation cloud at the focus (Lin et al. 2014; Vlaisavljevich et al. 2015; Khokhlova et al. 2011; Vlaisavljevich et al. 2013). Second, although the $-3 \mathrm{~dB}$ focal spots were well localised, there were lobes in the annulus and further the $-6 \mathrm{~dB}$ volume covered much of the disc; consequently given the stochastic nature of in vivo cavitation, which tends to be random and sparse (Coussios et al. 2007; Miller et al. 2000), it is likely that even if cavitation could be created, it would likely occur in multiple regions through the disc rather than just in the nucleus. Generating cavitation outside the focal region, 
e.g., in the annulus, has two negative consequences: it could lead to damage outside the target area and also shield of the nucleus preventing treatment in the target region (Coussios et al. 2007).

One strategy to overcome the cavitation control in this application would be to inject cavitation nuclei into the nucleus of the disc. For example, ultrasound contrast agents (UCAs) have been used in HIFU to induce cavitation enhanced therapy (McDannold et al. 2006; Tran et al. 2003). UCAs are probably not be appropriate for use in the IVD application because continuously multiple injections of UCAs would be needed for sustained cavitation due to the short life spans which could be even shorter due to the static overpressure in the IVD (Bouakaz et al. 2005; Chan et al. 2011). An alternative nucleation agent is a polymeric nanoparticle that has been developed in our group that traps gas in a pore which, in comparison to a UCA, has a tighter cavitation threshold and results in sustained cavitation activity in a tumour model in vivo for at least two minutes (Kwan et al. 2015). Preliminary experiments using these polymeric nanoparticles to mediate the fractionation of the nucleus in the disc from an ox tail showed sustained cavitation activity for at least 10 min of ultrasound exposure with the peak negative pressure of approximately $2 \mathrm{MPa}$ at $0.5 \mathrm{MHz}$ (Elbes et al. 2014) which we anticipate will be sufficient to fractionate a disc (Molinari. 2012).

Although placement of a needle is normally avoided in therapeutic ultrasound, in this specific application a needle will need to be employed to remove fractionated material and inject a biocompatible replacement polymer. Therefore we can make use of the needle access in two ways: first, a hydrophone (we anticipate using a fibre-optic hydrophone with a diameter of $100 \mu \mathrm{m}$ ) can be placed in the target region in the centre of the IVD in order to carry out the time-reversal process. Second, the needle could be used to inject of cavitation nuclei. In the simulations reported here, focal pressures ranging from 3.2 to $13 \mathrm{MPa}$ are achievable, which exceeds the $2 \mathrm{MPa}$ cavitation threshold of the polymeric nanoparticles in discs. Therefore by adjusting the amplitude of the ultrasound it should be possible to control the initiation of cavitation to regions where the pressure is above the cavitation threshold. Limiting the injection of the polymeric nanoparticles to the nucleus also means that the "hot spots" that occurred in the outer regions of the annulus should not result in cavitation as the threshold there will be the native tissue threshold which is a factor of five or more above the 
cavitation nuclei. This approach to spatial control of cavitation has been demonstrated using nanodroplets (Vlaisavljevich et al. 2013). The disc where this approach might not be successful is lumbar disc L1L2 where the $-3 \mathrm{~dB}$ regions extended from the nucleus to the annulus and warrants further investigation.

One variable that was not considered in this study was ultrasound frequency. We employed $500 \mathrm{kHz}$ partly because preliminary evidence (Molinari. 2012) suggested that it is effective in disc fractionation but also because given the propagation path it provides a trade-off between attenuation and aberration (which impact higher frequencies) and the ability to create tight focus (which is wavelength limited). Employing lower frequencies should in principal reduce the cavitation threshold; but the longer wavelengths would mean that the focal spot will increase in spatial extent. The increase in wavelength could be a particular issue in this application due to the presence of the bone surfaces above and below the discs potentially result in a waveguide like geometry.

One limitation of this study is that it is purely numerical and the soft tissue was segmented in to three homogeneous media: bone, fat, and other soft tissue. In real tissue the situation will be more complex, for example, the internal structure of the IVD was not included in the model as there was not sufficient contrast in the CT images. The limited data on the acoustic properties of the IVD tissue indicate that the outer rim has a higher sound speed $(1557-1597 \mathrm{~m} / \mathrm{s}$ vs $1553 \mathrm{~m} / \mathrm{s})$ and attenuation (1.24 Np/cm/MHz vs $0.21 \mathrm{~Np} / \mathrm{cm} / \mathrm{MHz}$ ) than the inner core (Molinari 2012; Tervonen 1988), perhaps due to the different water and collagen content in these two regions. One could employ other imaging modalities, e.g., MRI (Pfirrmann et al. 2001), to distinguish the internal structure of the disc however we anticipate that the differences in the acoustic properties are small enough that results reported here should not change dramatically. A second limitation is that only three patient models were used and in the future more models should be considered to ensure that the approach is feasible in a larger population. A third limitation is that the numerical model assumed linear acoustic propagation. For the acoustic parameters envisioned here we anticipate nonlinear distortion to occur in the propagation path. The shock formation distance for a $1 \mathrm{MPa}, 0.5 \mathrm{MHz}$ sinusoidal waveform in tissue is about 250 $\mathrm{mm}$ and so over the $170 \mathrm{~mm}$ focal length of the array harmonics should be generated even if a shock 
wave does not form. The nonlinear distortion of the waveform typically means that the negative pressure has a slightly lower gain than the linear simulations and the peak negative pressure may move slightly pre-focally (Canney et al. 2010). Despite these limitations we anticipate that the predicted pressure amplitudes and focusing presented here are sufficient that, even with some loss in performance, the concept will still be feasible. The pressure amplitudes predicted for the small and medium patient were at least 3 times larger than the threshold pressure required for the cavitation agents we would employ and so a small loss in focusing gain will still allow for the generation of cavitation.

\section{CONCLUSION}

The feasibility of extracorporeal ultrasound delivery to lumbar IVDs has been investigated through numerical simulations of linear acoustic propagation in three patients of different sizes. Ray-tracing was used to determine the acoustic windows to the IVD, which, in addition to anatomical restrictions (bowel gas and the spinal cord), suggested the placement of two rectangular arrays at the posteriorlateral sides of the patient. Time-reversal was employed to determining the phasing of the array elements by placing a spherical source in the target location and carrying out a full wave threedimensional back-propagation to determine the waveforms that would arrive at the elements of the two arrays. Time-reversal was shown to correct for the aberration of the sound beam resulting from bony structures and multiple tissue layers, resulting in a focus in the centre of the disc, for L2L3, L3L4 and L4L5 in most patients. However, the top-most lumbar disc L1L2 may not be a good candidate for treatment as other hot-spots existed in the disc. The results suggest that patient-specific pre-treatment planning is needed to determine if a disc is a suitable candidate. Although in principle the simulations could be used to determine the time-reversal signals for the array computationally, because the procedure associated with repair of the disc demands needle access to the disc we envision inserting either an ultrasound source or hydrophone in the centre of the disc in order that physical time-reversal can be carried out which should be more robust than employing a numerical simulation. For cases where a focus was achieved the simulations predict for realistic source 
pressures that focal pressures should exceed $3 \mathrm{MPa}$ which although not high enough to fractionate

336 tissue directly would be sufficient to initiate cavitation in the presence of cavitation nuclei which

337 would also assist in keeping the cavitation activity localised in the target region.

\section{ACKNOWLEDGEMENTS}

339 This research is supported by EPSRC through the projects EP/K021729/1 and EPSRC EP/K02020X/1.

340 The authors would like to thank Dr Gerry Harvey and Robbie Banks from Weidlinger Associates, Ltd.

341 (PZFlex Europe) for their help on the optimization of the models. Data supporting this manuscript

342 will be made available on the Oxford University Research Archive. 


\section{REFERENCES:}

Al-Bataineh O, Jenne J, Huber P. Clinical and future applications of high intensity focused ultrasound in cancer. Cancer Treat Rev 2012;38:346-353.

Aubry J-F, Pernot M, Marquet F, Tanter M, Fink M. Transcostal high-intensity-focused ultrasound: ex vivo adaptive focusing feasibility study. Phys Med Biol 2008;53:2937.

Bobkova S, Gavrilov L, Khokhlova V, Shaw A, Hand J. Focusing of high-intensity ultrasound through the rib cage using a therapeutic random phased array. Ultrasound Med Biol 2010;36:888-906.

Bouakaz A, Versluis M, de Jong N. High-speed optical observations of contrast agent destruction. Ultrasound Med Biol 2005;31:391-399.

354 Canney MS, Khokhlova VA, Bessonova OV, Bailey MR, Crum LA. Shock-Induced Heating and 355 Millisecond Boiling in Gels and Tissue Due to High Intensity Focused Ultrasound. Ultrasound Med 356 Biol 2010;36:250-267.

357 Chan SC, Ferguson SJ, Gantenbein-Ritter B. The effects of dynamic loading on the intervertebral disc. Eur Spine J 2011;20:1796.

Cheung KM, Karppinen J, Chan D, Ho DW, Song Y-Q, Sham P, Cheah KS, Leong JC, Luk KD. Prevalence and pattern of lumbar magnetic resonance imaging changes in a population study of one thousand forty-three individuals. Spine 2009;34:934-940.

Coric D, Mummaneni PV. Nucleus replacement technologies. 2008; cancer treatment by high-intensity focused ultrasound (HIFU). Int J Hyperthermia 2007;23:105-120. 
Di Martino A, Vaccaro AR, Lee JY, Denaro V, Lim MR. Nucleus pulposus replacement: basic science and indications for clinical use. Spine 2005;30:S16-S22.

Elbes D, Boubriak O, Qiao S, Molinari M, Urban J, Cleveland R, Coussios C. Ultrasound-induced

fractionation of the intervertebral disk. J Acoust Soc Am 2014;136:2281-2281.

Fink M. Time reversal of ultrasonic fields. I. Basic principles. IEEE Trans Ultrason Ferroelectr Freq Control 1992;39:555-566.

Fink M, Montaldo G, Tanter M. Time-reversal acoustics in biomedical engineering. Annu Rev Biomed Eng 2003;5:465-497.

Gateau J, Marsac L, Pernot M, Aubry J-F, Tanter M, Fink M. Transcranial Ultrasonic Therapy Based on Time Reversal of Acoustically Induced Cavitation Bubble Signature. IEEE Trans Biomed Eng 2010;57:134-144.

Hoy D, March L, Brooks P, Blyth F, Woolf A, Bain C, Williams G, Smith E, Vos T, Barendregt J. The global burden of low back pain: estimates from the Global Burden of Disease 2010 study. Ann Rheum Dis 2014;73:968-974.

Khokhlova TD, Canney MS, Khokhlova VA, Sapozhnikov OA, Crum LA, Bailey MR. Controlled tissue emulsification produced by high intensity focused ultrasound shock waves and millisecond boiling. J Acoust Soc Am 2011;130:3498-3510. Ultrasound-propelled nanocups for drug delivery. Small 2015;11:5305-5314. technology for prostatic tissue ablation in an in vivo canine model. Urology 2008;72:682-686. 
Lewis G. Nucleus pulposus replacement and regeneration/repair technologies: present status and future prospects. J Biomed Mater Res B Appl Biomater 2012;100:1702-1720.

Lin K-W, Kim Y, Maxwell AD, Wang T-Y, Hall TL, Xu Z, Fowlkes JB, Cain C. Histotripsy beyond the intrinsic cavitation threshold using very short ultrasound pulses: Microtripsy. IEEE Trans Ultrason Ferroelectr Freq Control 2014;61:251-265.

Maniadakis N, Gray A. The economic burden of back pain in the UK. Pain 2000;84:95-103.

Marquet F, Pernot M, Aubry J-F, Montaldo G, Marsac L, Tanter M, Fink M. Non-invasive transcranial ultrasound therapy based on a 3D CT scan: protocol validation and in vitro results. Phys Med Biol 2009;54:2597-2613.

McDannold NJ, Vykhodtseva NI, Hynynen K. Microbubble contrast agent with focused ultrasound to 396 create brain lesions at low power levels: MR imaging and histologic study in rabbits. Radiology 2006;241:95-106.

Medel R, Monteith SJ, Elias WJ, Eames M, Snell J, Sheehan JP, Wintermark M, Jolesz FA, Kassell NF. Magnetic resonance-guided focused ultrasound surgery: Part 2: A review of current and future applications. Neurosurgery 2012;71:755-763.

401

Miller DL, Kripfgans OD, Fowlkes JB, Carson PL. Cavitation nucleation agents for nonthermal

402 ultrasound therapy. J Acoust Soc Am 2000;107:3480-3486.

403

Molinari MB. Mechanical fractionation of the intervertebral disc. DPhil thesis, University of Oxford 404 2012; 

brain surgery with an ultrasonic time reversal mirror. J Neurosurg 2007;106:1061-1066.

Pfirrmann CW, Metzdorf A, Zanetti M, Hodler J, Boos N. Magnetic resonance classification of lumbar intervertebral disc degeneration. Spine 2001;26:1873-1878.

Qiao S, Shen G, Bai J, Chen Y. Transcostal high-intensity focused ultrasound treatment using phased array with geometric correction. J Acoust Soc Am 2013;134:1503-1514. 74.

Qiao S, Jackson E, Coussious C-C, Cleveland RO. Simulation of nonlinear propagation of biomedical ultrasound using PZFlex and the KZK Texas code, J. Acoust. Soc. Am. 2016; 140: 2039-2046. noninvasive technology for controlled tissue ablation (histotripsy) in the rabbit kidney. J Urol 2006;175:734-738.

Shaheed CA, Maher CG, Williams KA, McLachlan AJ. Interventions available over the counter and advice for acute low back pain: systematic review and meta-analysis. J Pain 2014;15:2-15. among primary care physicians, the general population and patients at risk - the Shape of the Nations survey . Current Medical Research and Opinion 2007; 23:1, 29-47. ter Haar Gail, Coussios C. High intensity focused ultrasound: physical principles and devices. Int J Hyperthermia 2007;23:89-104. Neuroorthopedics 1988;5:67-73. 
Thomas J-L, Fink MA. Ultrasonic beam focusing through tissue inhomogeneities with a time reversal mirror: application to transskull therapy. IEEE Trans Ultrason Ferroelectr Freq Control 1996;43:11221129.

Tran BC, Seo J, Hall TL, Fowlkes JB, Cain CA. Microbubble-enhanced cavitation for noninvasive ultrasound surgery. IEEE Trans Ultrason Ferroelectr Freq Control 2003;50:1296-1304.

Vlaisavljevich E, Durmaz YY, Maxwell A, ElSayed M, Xu Z. Nanodroplet-Mediated Histotripsy for Image-guided Targeted Ultrasound Cell Ablation. Theranostics 2013;3:851-864.

Vlaisavljevich E, Lin K-W, Maxwell A, Warnez MT, Mancia L, Singh R, Putnam AJ, Fowlkes B, Johnsen E, Cain C, Xu Z. Effects of Ultrasound Frequency and Tissue Stiffness on the Histotripsy Intrinsic Threshold for Cavitation. Ultrasound Med Biol 2015;41:1651-1667.

Walker BF. The prevalence of low back pain: a systematic review of the literature from 1966 to 1998. Clin Spine Surg 2000;13:205-217.

Weeks EM, Platt MW, Gedroyc W. MRI-guided focused ultrasound (MRgFUS) to treat facet joint osteoarthritis low back pain-case series of an innovative new technique. Eur Radiol 2012;22:28222835.

Willems PC, Staal JB, Walenkamp GH, de Bie RA. Spinal fusion for chronic low back pain: systematic review on the accuracy of tests for patient selection. Spine J 2013;13:99-109. cavitational ultrasound therapy-histotripsy. IEEE Trans Ultrason Ferroelectr Freq Control 2008;55. 
449 Figure 1. A schematic of the vertebral joint. Here it is partly cut away to show the annulus fibrosus

450 (AF) surrounding the nucleus pulposus (NP) of the intervertebral disc, the cartilagenous endplate

451 (CEP) and bony endplate (BEP) interspersed between the disc and vertebral body (VB), and the spinal

452 canal (SC) lying behind the vertebral bodies and the disc. The spinal canal, running between the discs

453 and the spinal processes (SP) and apophyseal joints, encloses the spinal cord which gives rise to the

454 nerve roots (NR), running adjacent to the posterior portion of the disc. (adapted from Urban and

455 Roberts 1986)

456 Figure 2: (a) The diagram of the steps of modelling procedure: CT images are segmented into tissue

457 types, lumbar discs are identified and target locations set manually, data sets are imported into

458 acoustic software and ray tracing is carried out, the array placement is determined for full wave

459 ultrasound simulations. Representative two dimensional slices through each of the three segmented

460 data sets are shown for model I (b), II (c) and III (d).

461 Figure 3. Top: An example of the ray-tracing results showing rays cast from the target location that do

462 not intersect with bone. The contour shows the boundary at which no line-of-sight rays exist. Bottom:

463 The pressure distribution on a spherical surface with a radius of $170 \mathrm{~mm}$, as calculated by PZflex, and

464 overlayed is the contour of the acoustic window as calculated from the ray-tracing method.

Figure 4. The simulation results of ultrasound delivery in the L4L5 disc of model II with (a) uniform transmitting signals and (b) time-reversed signals. The white contours show the bone, the black contours the estimated edge of the IVD, and the white stars the desired target location.

Figure 5. Simulated pressure gain distribution in the cross-section plane of all four lumbar discs. The thick black contours indicate the estimated shape of discs. (a) model I, (b) model II, (c) model III.

Figure 6. (a) focal pressure gain (normalised by source pressure) and (b) offset of focus (distance between the target and the location of the peak pressure) for all four discs and three patients. An offset is not reported for L4L5 in model III as a focal spot was not generated in this case. 
473 Figure 7. The $-3 \mathrm{~dB}$ (red contours) and $-6 \mathrm{~dB}$ area (blue contours) of the pressure field in all the discs.

474 The thick black contours indicate the estimated shape of discs and the thin black contours indicate the 475 estimated area of the nucleus. (a) model I, (b) model II, (c) model III. 


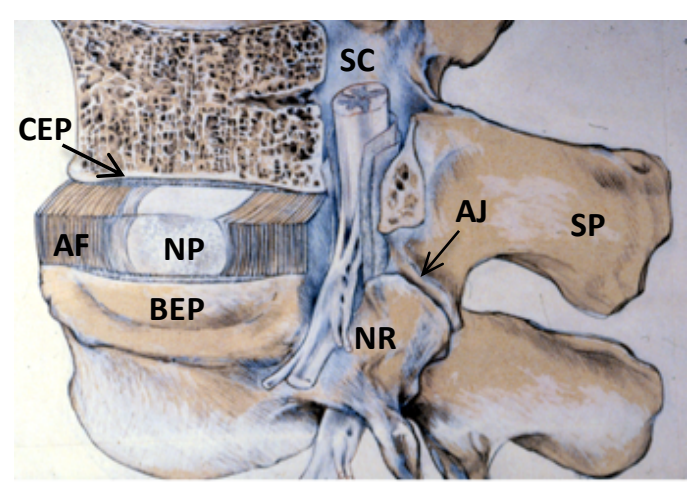

Figure 1. A schematic of the vertebral joint. Here it is partly cut away to show the annulus fibrosus (AF) surrounding the nucleus pulposus (NP) of the intervertebral disc, the cartilagenous endplate (CEP) and bony endplate (BEP) interspersed between the disc and vertebral body (VB), and the spinal canal (SC) lying behind the vertebral bodies and the disc. The spinal canal, running between the discs and the spinal processes (SP) and apophyseal joints, encloses the spinal cord which gives rise to the nerve roots (NR), running adjacent to the posterior portion of the disc. (adapted from Urban and Roberts 1986). 

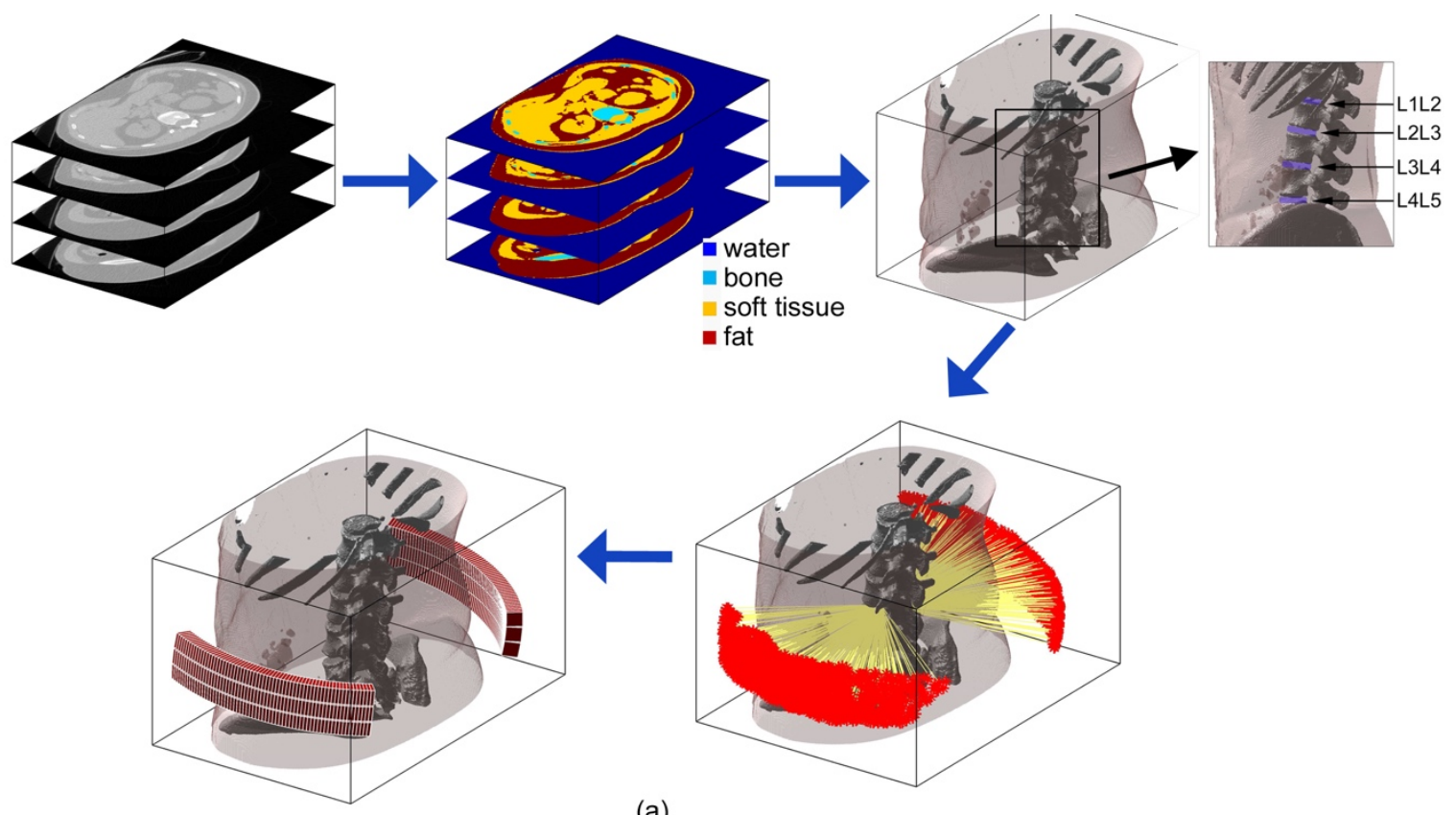

(a)

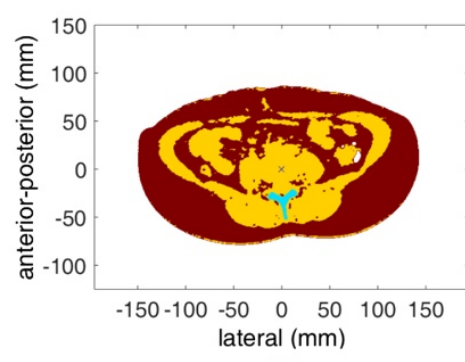

(b)

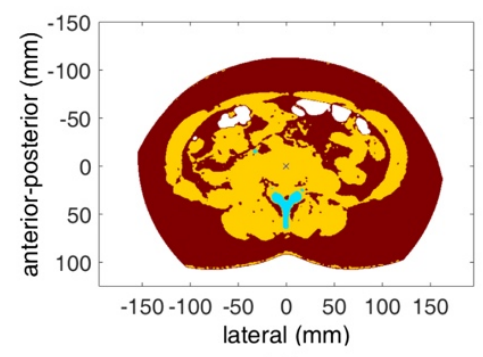

(c)

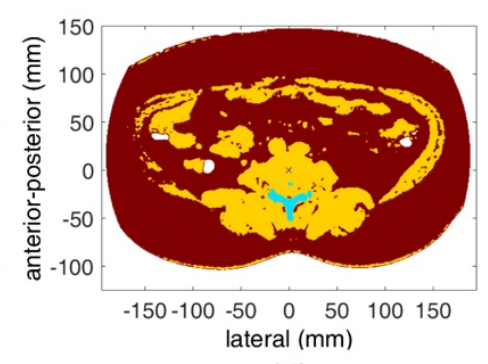

(d)

Figure 2: (a) The diagram of the steps of modelling procedure: CT images are segmented into tissue types, lumbar discs are identified and target locations set manually, data sets are imported into acoustic software and ray tracing is carried out, the array placement is determined for full wave ultrasound simulations. Representative two dimensional slices through each of the three segmented data sets are shown for model I (b), II (c) and III (d). 

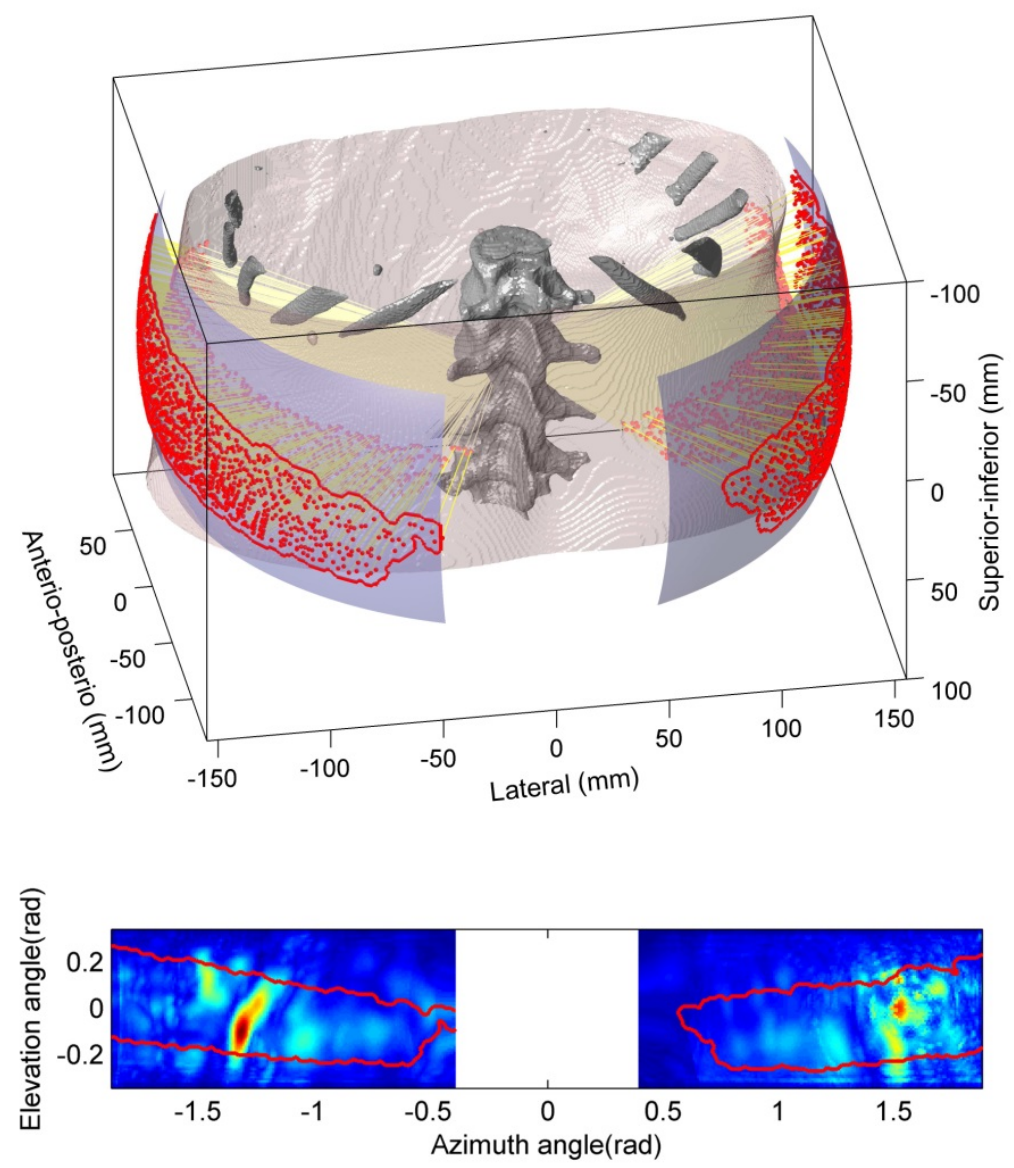

Figure 3. Top: An example of the ray-tracing results showing rays cast from the target location that do not intersect with bone. The contour shows the boundary at which no line-of-sight rays exist. Bottom: The pressure distribution on a spherical surface with a radius of $170 \mathrm{~mm}$, as calculated by PZflex, and overlayed is the contour of the acoustic window as calculated from the ray-tracing method. 

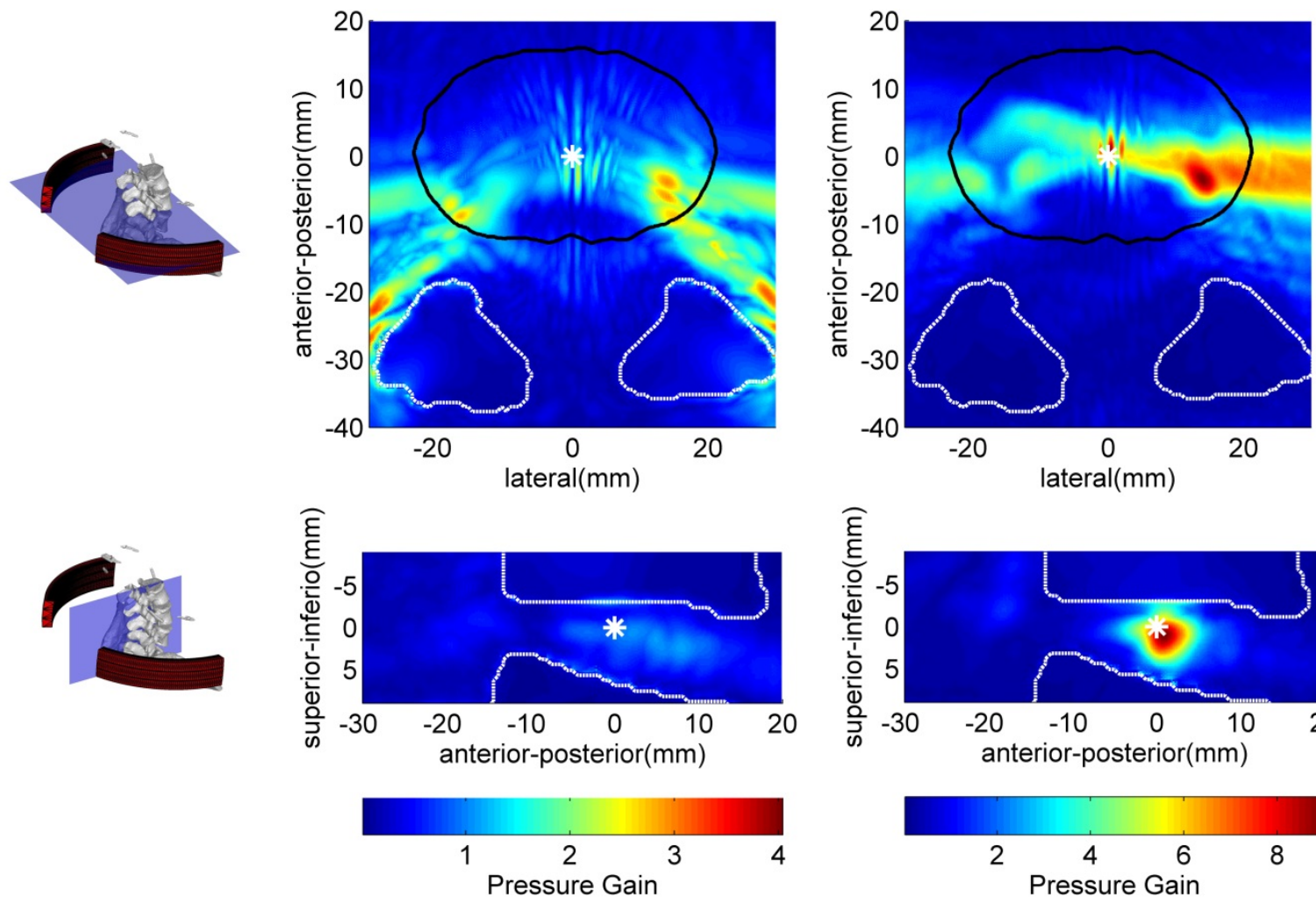

(a)

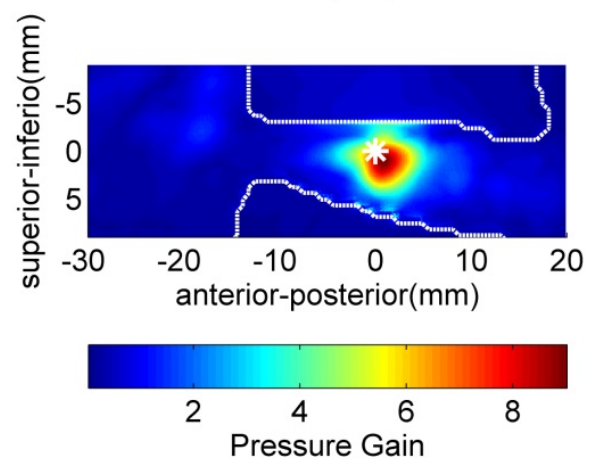

(b)

Figure 4. The simulation results of ultrasound delivery in the L4L5 disc of model II with (a) uniform transmitting signals and (b) time-reversed signals. The white contours show the bone, the black contours the estimated edge of the IVD, and the white stars the desired target location. 

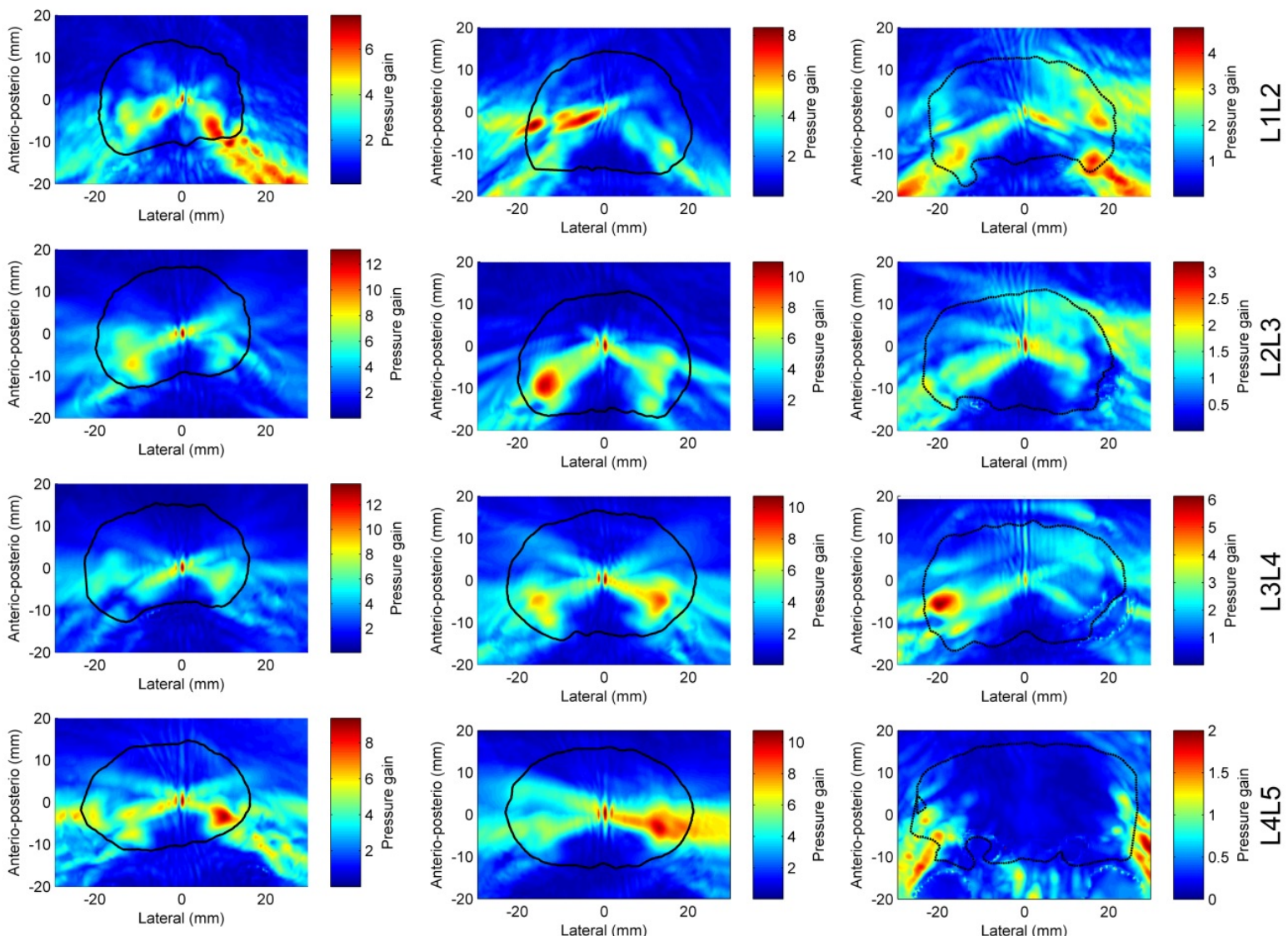

(a)

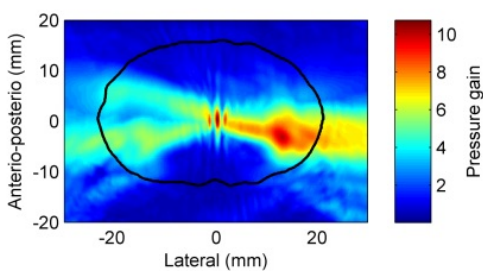

(b)

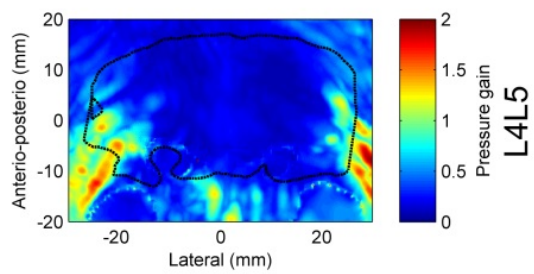

(c)

Figure 5. Simulated pressure gain distribution in the cross-section plane of all four lumbar discs. The thick black contours indicate the estimated shape of discs. (a) model I, (b) model II, (c) model III. 


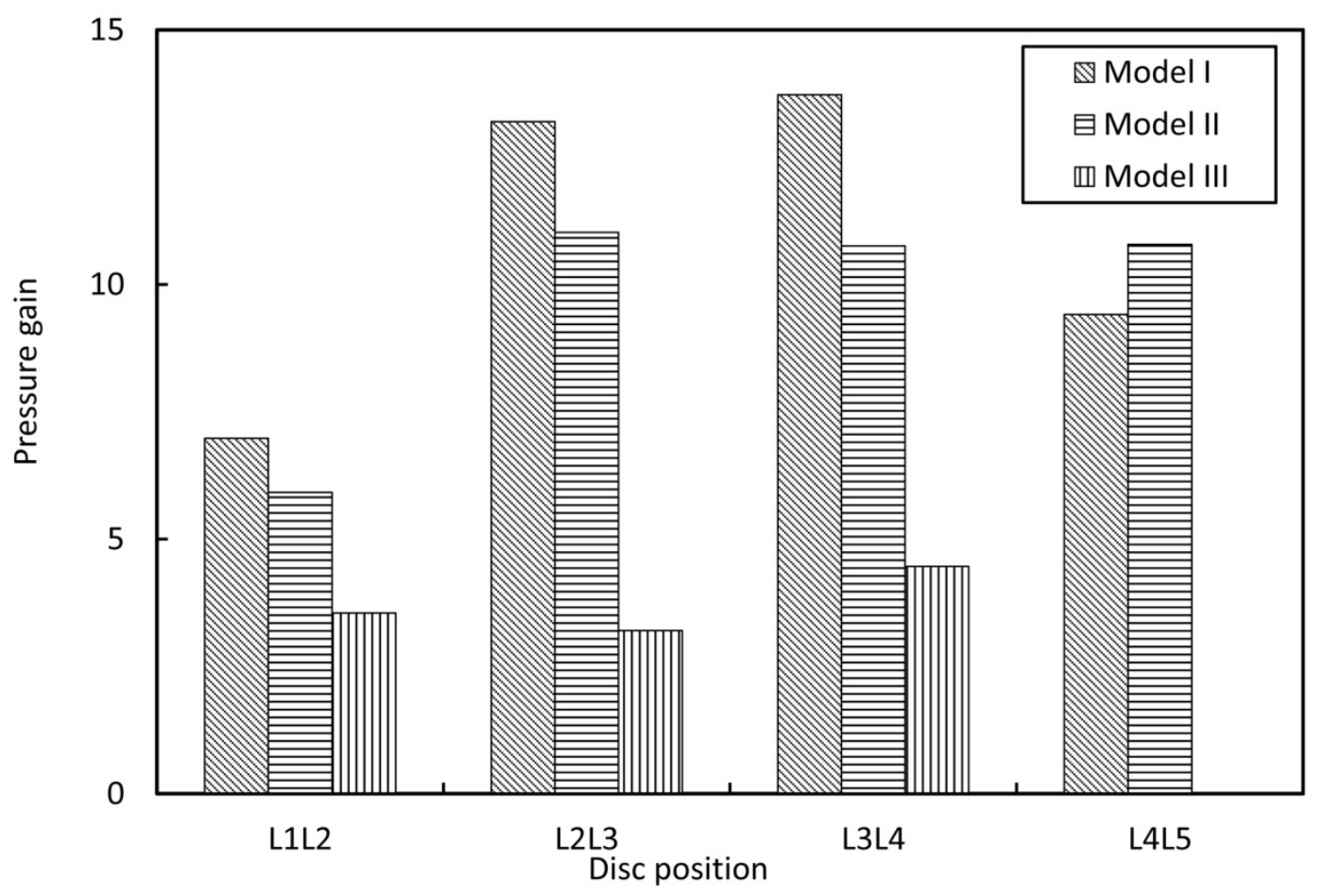

(a)

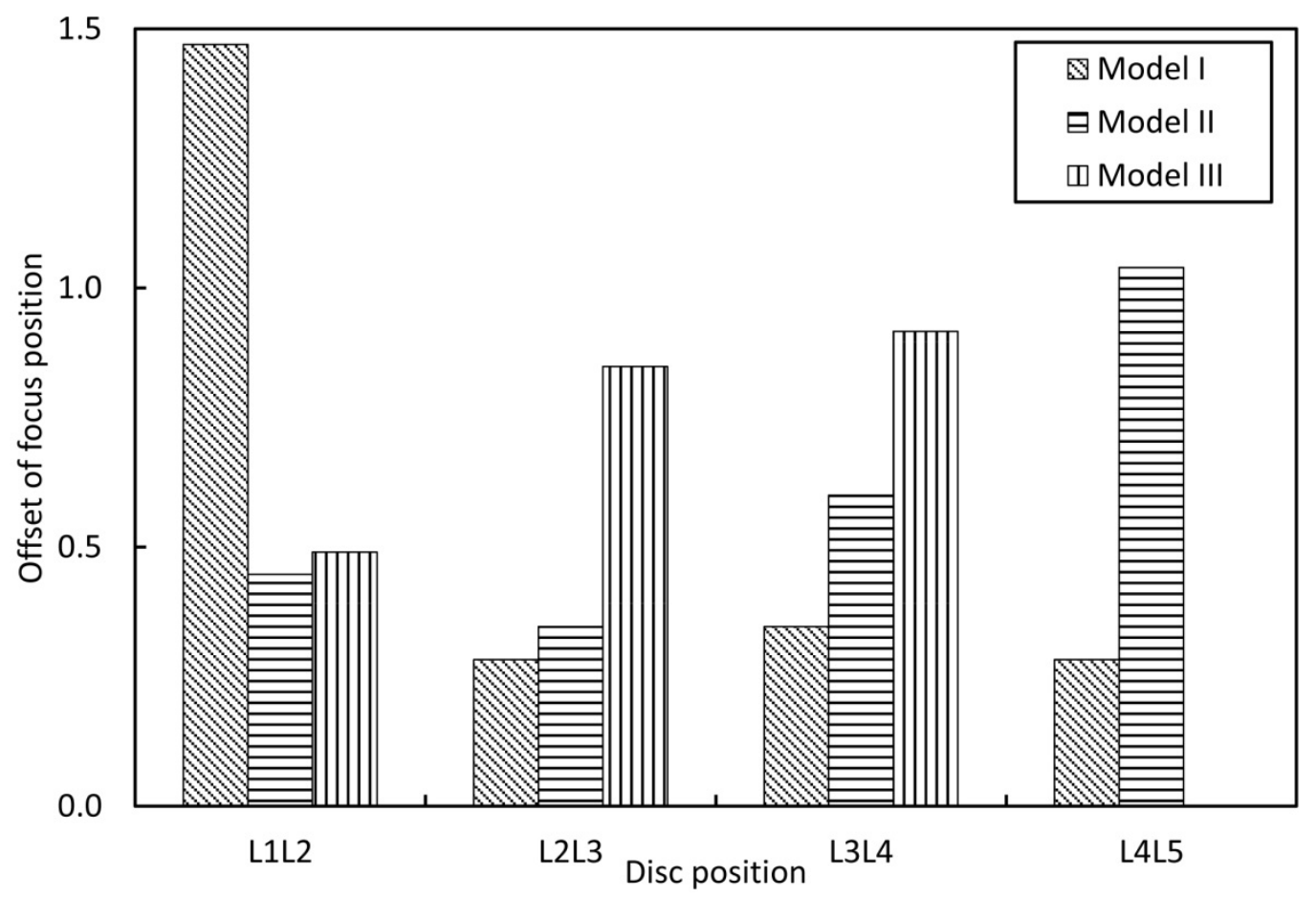

(b)

Figure 6. The focal pressure gain (a) and the spatial offset between the peak pressure and the desired focus (b) for all four discs and three patients. There is no offset for L4L5 in model III as a focal spot was not generated in this case. 

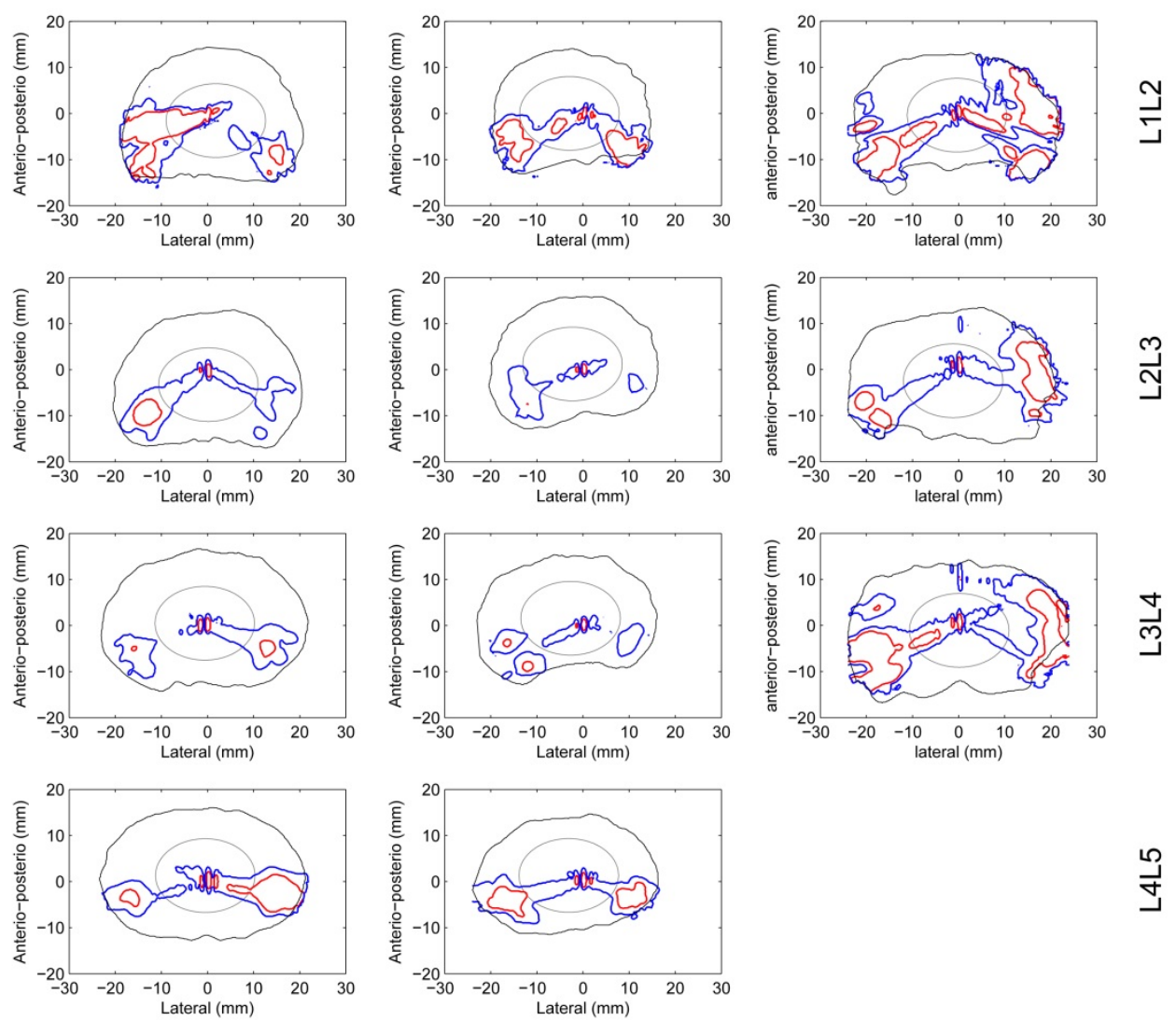

(b)

곧

มิ

$\stackrel{?}{\exists}$

(a)

(c)

Figure 7. The $-3 \mathrm{~dB}$ (red contours) and $-6 \mathrm{~dB}$ area (blue contours) of the pressure field in all the discs. The thick black contours indicate the estimated shape of discs and the thin black contours indicate the estimated area of the nucleus. (a) model I, (b) model II, (c) model III. 